\title{
Disastrous Failure of a Carbide-Studded Cutting Disc
}

Michael Panzenböck, *

* Department of Physical Metallurgy and Materials Testing, University of Leoben, A-8700 Leoben, Austria

Special cutting discs make it possible to trench soft as well as hard materials. The heat input can be reduced significantly if the cutting disc and the material are co-ordinated. Parameters which influence this system are cutting material (grain size) and binders, cutting speed and cooling fluid.

Cutting discs for brickwork, concrete, flagging, etc. normally consist of cheap carrier material (e.g. carbon steel or low alloyed steels) and this base is assembled with special cutting segments at the circumference. Cutting segments are very often cemented carbides with varying content of industrial diamonds. Diameters of cutting discs range between a few centimetres up to one metre. According to the diameter the revolution speed varies from a few $100 \mathrm{rpm}$ up to $8500 \mathrm{rpm}$.

In this case of damage four cutting segments disassociated from the disc when the cutting machine reached its operating speed of $6600 \mathrm{rpm}$. Eyes and face of the worker were severely injured by the split segments. Figure 1.a shows the disc with the missing segments. Moreover, no additional damage is visible neither on the front side nor on the back side of the disc nor on the remaining segments (Fig. 1b). Latter suggests that there was no contact between cutting tool and concrete or brick wall. To clarify the circumstances which led to the accident SEM investigations (Scanning Electron Microscopy) and microanalysis were done. Furthermore tension tests (with a tensile testing equipment) were carried out to measure the adhesive strength between the remaining segments (Fig. $1 \mathrm{a}, 5 \mathrm{~b}-16 \mathrm{~b})$ and the carrier material. For determining the way of joining between cutting segment and carrier material small specimens were cut out of the disc. These samples were broken in liquid nitrogen. Investigations of the fracture surface were carried out, too.

Tensile tests exhibited that the force to detach the segments from the disc ranged from $310 \mathrm{~N}$ to 2760 N. Calculating the acting centrifugal force from the given diameter (see Fig. 1a), the weight of a segment $(3.735 \mathrm{~g})$ and the rotation speed of $6600 \mathrm{rpm}$ shows that the force is about $200 \mathrm{~N}$. The calculated load is near the lowest adhesive force $(310 \mathrm{~N})$ measured before. Consequently the safety factor is about 1.5 .

Figure 2a shows the specimen cut out of the cutting disc. Within the cutting segment which is part of the specimen diamond particles (dark spots in Fig. 2a) with smooth surfaces are visible. Furthermore a gap between carrier material and cutting segment can clearly be seen. The joint area exhibits a rippled surface especially on the carrier material (compare Fig. 2b). After pulling off the cutting segment two different surface topographies appear on the carrier material. Basically there is the rippled surface (Fig. 3a) but few areas reveal typical shear structures (see Fig. 3b). In both cases the surface consists of $\mathrm{Cu}$ and $\mathrm{Sn}$, which was proved by chemical analysis. Both the rippled surface topography and the chemical composition point out that a soldering process with tin bronze was used to join the cutting segment and the steel disc. Further investigations of the surface of the steel disc reveal a thin copper layer with a few microns of thickness. This layer is important for soldering.

Summing up the disastrous failure was due to an inadequate brazing process. 


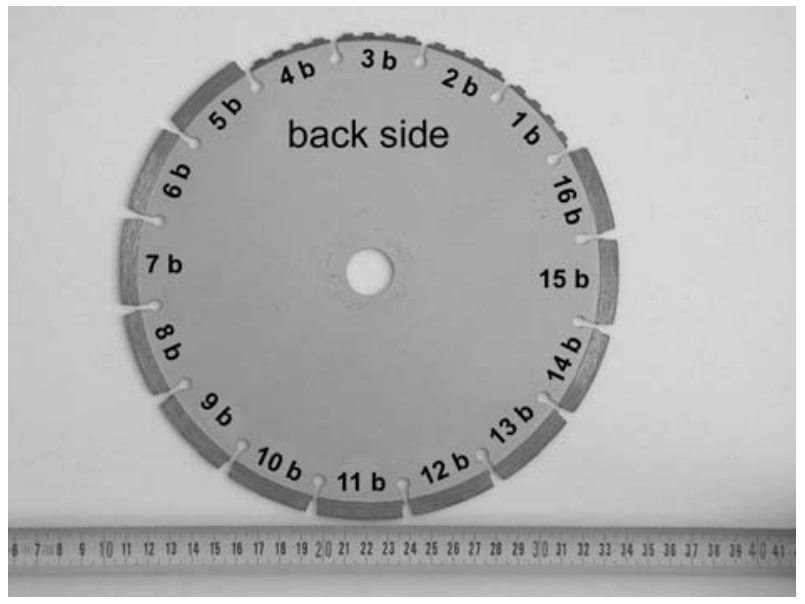

FIG. 1a. Overview of the cutting wheel

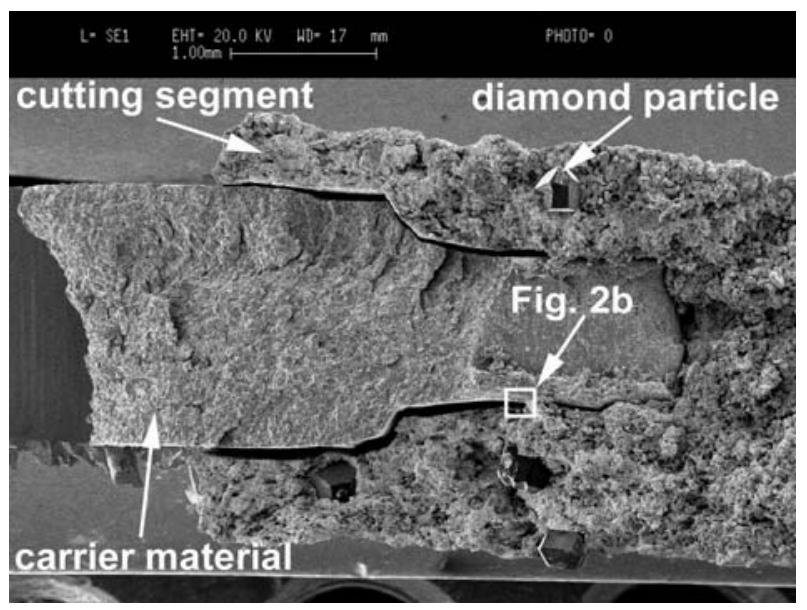

FIG. 2a. Broken part of the cutting wheel

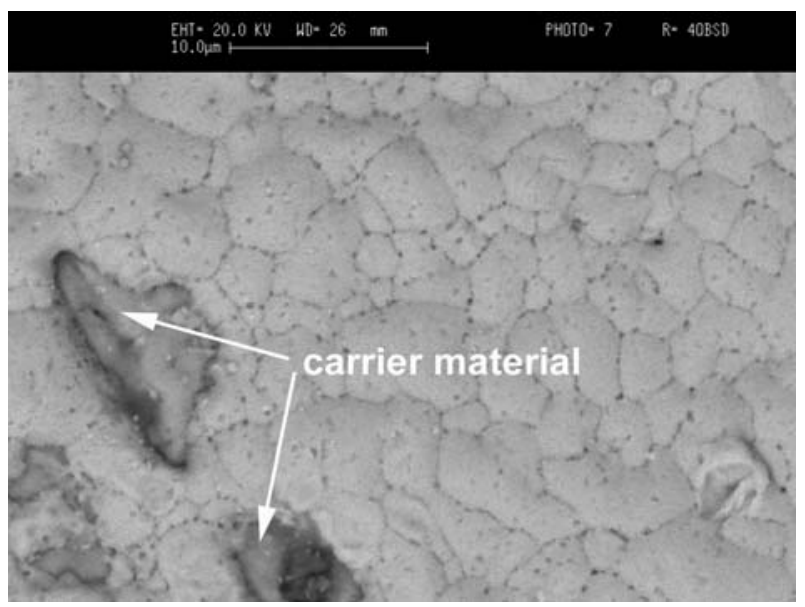

FIG. 3a. Rippled surface structure of the brazing solder (phase contrast)

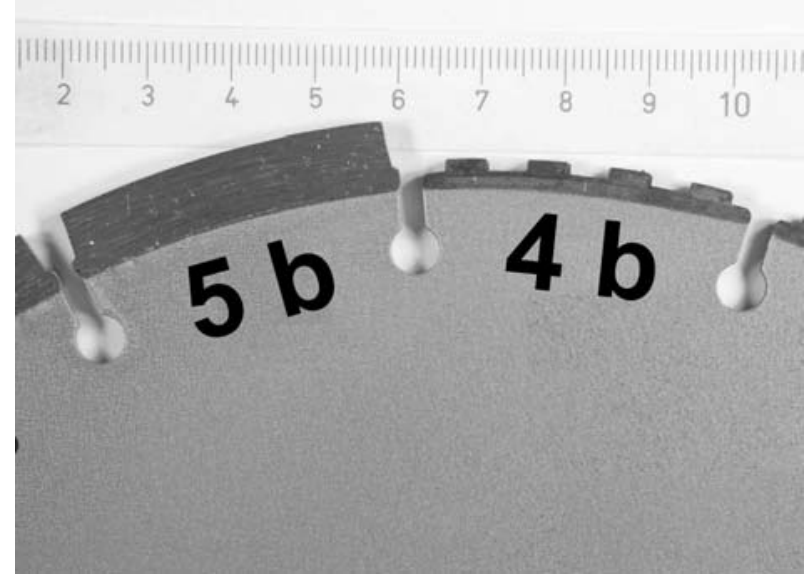

FIG. 1b. Detail of Fig. 1a

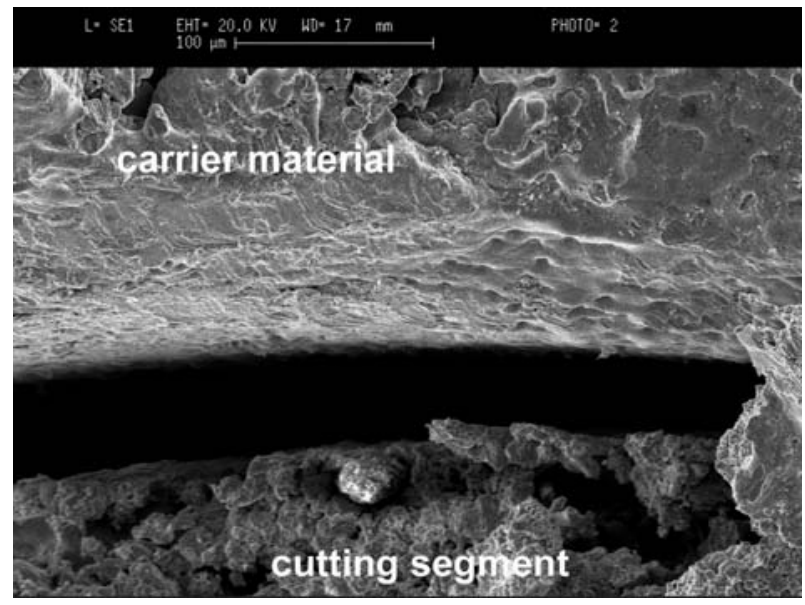

FIG. 2b. Detail of Fig. 2a

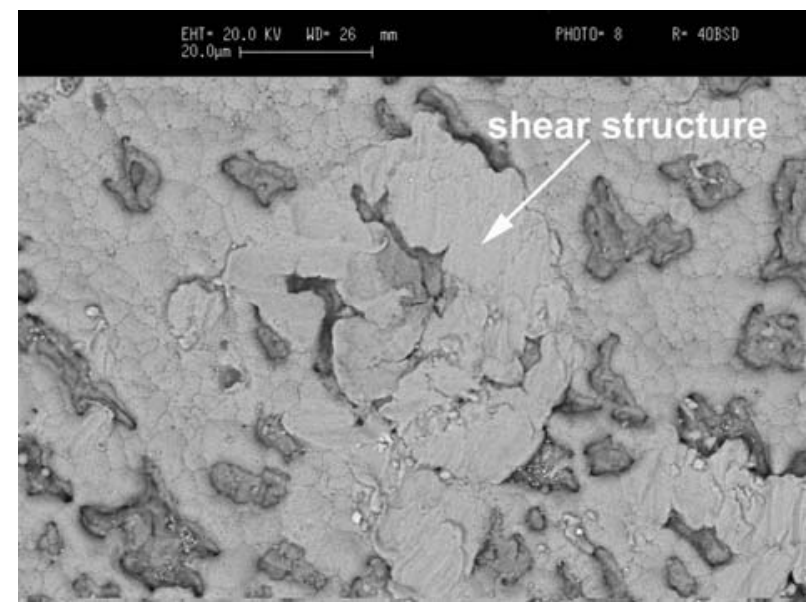

FIG. 3b. Rippled surface structure and shear zones of the brazing solder (phase contrast) 